\title{
Comparing The Efficacy of Sevoflurane To Propofol For Inflammatory Response During Venovenous Extracorporeal Membrane Oxygenation
}

\section{Rongzhi Zhang}

Lanzhou University Second Hospital, Lanzhou University Jian Huang

Lanzhou University Second Hospital, Lanzhou University Jianbao Yang Lanzhou University Second Hospital, Lanzhou University

\section{Xingdong Cheng}

Lanzhou University Second Hospital, Lanzhou University

\section{Kerong Zhai}

Lanzhou University Second Hospital, Lanzhou University

Shilin Wei

Lanzhou University Second Hospital, Lanzhou University

Jian Li

Lanzhou University Second Hospital, Lanzhou University

Yongnan Li ( $\nabla$ lyngyq2006@foxmail.com )

Lanzhou University Second Hospital, Lanzhou University

\section{Bingren Gao}

Lanzhou University Second Hospital, Lanzhou University

\section{Research Article}

Keywords: Acute respiratory distress syndrome, Venovenous extracorporeal membrane oxygenation, sevoflurane, propofol, rat model

Posted Date: December 3rd, 2021

DOI: https://doi.org/10.21203/rs.3.rs-1120705/v1

License: (c) (1) This work is licensed under a Creative Commons Attribution 4.0 International License. Read Full License 


\section{Abstract}

Background: Acute respiratory distress syndrome (ARDS), a clinical syndrome resulting from intrapulmonary and/or extrapulmonary causes, is characterized by refractory hypoxemia. Currently, venovenous extracorporeal membrane oxygenation (VV ECMO) is considered as a reasonable alternative to salvage ARDS, but it requires sedation of the patient. Several studies suggest that anesthetics, such as sevoflurane and propofol, have lung protective and immunomodulatory functions. The aim of this study was to explore the effects of sevoflurane and propofol on ARDS in VV ECMO rat models.

Methods: To establish the ARDS model, male Sprague-Dawley (SD) rats were injected with $100 \mathrm{mg} / \mathrm{kg}$ oleic acid (OA). Twenty-four SD rats were randomly divided into two groups: sevoflurane group (Sevo group) and propofol group (Pro group). The basic vital signs of rats in each group were continuously monitored using a life monitor, and arterial blood gas tests were performed at the following three time points: T0 (baseline), T1 (the time to ARDS), and T2 (after weaning from ECMO for $1 \mathrm{~h}$ ). Bicinchoninic acid assay (BCA) method was used to determine protein concentration in bronchial alveolar lavage fluid (BALF), whereas hemotaxylin and eosin (HE) staining was used to evaluate the lung pathological scores in each group. In addition, inflammatory factors (IL-1b, TNF-a, and MPO) in BALF, serum, and lung were determined using enzyme-linked immunosorbent assay (ELISA) and immunohistochemistry (IHC).

Results: With regard to the blood gas index, the Sevo group exhibited a better effect in improving the oxygenation function than the Pro group $(P<0.05)$. However, there was no significant difference in mean arterial pressure (MAP) between the two groups ( $P>0.05$ ). After VV ECMO assistance, the degree of lung injury and inflammatory changes in the Sevo group were significantly reduced compared to the Pro group.

Conclusion: This study shows that sedation with sevoflurane during VV ECMO assisted ARDS in rats improved lung injury and inflammation, and was better than propofol in improving the oxygenation function.

\section{Introduction}

Acute respiratory distress syndrome (ARDS) is a common condition encountered in the intensive care unit (ICU), with about $10 \%$ of all patients admitted to the ICU developing ARDS [1]. The Extracorporeal Life Support Organization guideline proposed that extracorporeal membrane oxygenation (ECMO) is an effective treatment for patients suffering from severe ARDS [2]. Venovenous extracorporeal membrane oxygenation (VV ECMO) is a rescue therapy used in severe ARDS patients to allow lung protective mechanical ventilation and provide adequate time for treating the cause of ARDS, thereby permitting lung healing $[3,4]$. However, some ARDS patients require deep sedation during the early phase of admission [5]. It is worth noting that the primary goal of sedation for patients receiving lung protective ventilation strategy is to provide comfort and safety [6]. Patients receiving VV ECMO may require higher sedation compared to ARDS patients without VV ECMO in order to tolerate the invasiveness of the procedure, 
compensate for sedatives consumed by the ECMO circuit itself, and because patients who receive ECMO tend to be younger and have higher illness severity $[4,7,8]$.

Evidence suggests that the desired depth of sedation dictates the sedative choice [9]. For mechanicallyventilated adults, guidelines recommend analgesia-first, and if continuous sedation is required, propofol is the ideal agent [10]. When patients do not require deep sedation, sevoflurane will induce light-to-deep sedation [11]. In addition, accumulating evidence has shown that propofol and sevoflurane have antiinflammatory effects $[12,13]$. Moreover, a previous study found that the inflammatory response in ventilated patients undergoing lung resection was significantly suppressed by sevoflurane than propofol [14]. Propofol was also shown to inhibit neutrophil and IL-8 responses in a rabbit ARDS model [15].

Ferrando et al. revealed that sevoflurane ameliorated the lung inflammatory response more than propofol in ARDS model pigs [16]. However, the effect of these anesthetic agents on the inflammatory response in ARDS patients during VV ECMO has not yet been evaluated.

Given the unique physiological characteristics of VV ECMO, the above findings of sevoflurane and propofol in non-VV ECMO patients are not generally applicable to this process. Therefore, this study used the previously established VV ECMO rat model to explore the anti-inflammatory response effect of inhaled sevoflurane and intravenous propofol during VV ECMO. The findings provide a valuable theoretical basis for clinical application.

\section{Methods}

All protocols were approved by the Lanzhou University Second Hospital (Lanzhou, Gansu, China) animal experimental ethics committee (approval number: D2021-020) and were in accordance with the animal care guidelines of the National Institutes of Health and Animal Research: Reporting of In Vivo Experiments (ARRIVE) guidelines. All efforts were made to minimize suffering and to reduce the number of rats used.

\section{Animals}

Experiments were performed in male Sprague-Dawley (SD) rats aged 12 weeks, weighing $350 \pm 50 \mathrm{~g}$ and purchased from Lanzhou Veterinary Research Institute, Chinese Academy of Sciences (Lanzhou, Gansu, China). All rats were housed in standard conditions, with a 12-h light/dark cycle, and ad-libitum access to food and water. Notably, all experiments were performed during the light cycle (from 9:00 am to 5:00 pm).

\section{The experimental protocol}

Animals were randomly assigned to two experimental groups ( $n=12$ for each group): sevoflurane (Sevo) group and propofol (Pro) group. Rats were first anesthetized with $1 \%$ pentobarbital sodium $50 \mathrm{mg} / \mathrm{kg}$ intraperitoneally, followed by fixing on the anatomical operating table in the supine position. Next, endotracheal intubation with a $16 \mathrm{G}$ needle was performed under direct vision and animals were connected to a rodent ventilator (Shanghai Alcott Biotech Co. Ltd., China). According to a previous study, 
the ventilator parameters were set as follows: tidal volume $6 \mathrm{ml} / \mathrm{kg}$, respiratory rate $70-75 \mathrm{breaths} / \mathrm{min}$, positive end-expiratory pressure (PEEP) $2 \mathrm{cmH}_{2} \mathrm{O}$, and inhalation/exhalation ratio (I:E) 1:2. An electronic thermometer was placed in the anus to monitor the body temperature, and the temperature of the rats was maintained at $36 \pm 0.5^{\circ} \mathrm{C}$ using a heating lamp. Notably, the right femoral artery and vein were exposed and free under local anesthesia with 1\% lidocaine. A 24G intravenous indwelling needle was punctured into the femoral artery and a monitor was connected for continuous hemodynamic monitoring. ARDS was then induced by intravenous injection of $100 \mathrm{mg} / \mathrm{kg}$ oleic acid (OA) (99\%, Sigma-Aldrich, USA) as previously described. The arterial blood gas was measured $1 \mathrm{~h}$ after OA injection, and the $\mathrm{PaO}_{2} / \mathrm{FiO}_{2} \leq 300$ was used as the model standard of ARDS. The VV ECMO rat model was established as previously reported [17]. After VV ECMO running, Sevo group rats inhaled 2\% sevoflurane (Maruishi Pharmaceutical Co. Ltd., Japan), whereas Pro group rats were infused with 1\% propofol $500 \mu \mathrm{g} \cdot \mathrm{kg}-1 \cdot \mathrm{min}-1$ (Corden Pharma S.P.A., USA). Notably, the whole experiment lasted for 3 h. Figure 1 shows the flow diagram of the experimental protocol.

\section{Arterial blood gas analysis (BGA) and collection of bronchoalveolar lavage fluid (BALF)}

Blood was collected from the femoral artery, and BGA was performed at the following three time points: T0 (baseline), T1 (the time to ARDS), and T2 (after weaning from ECMO for $1 \mathrm{~h}$ ). At the end of the experiment, the left hilum was clipped with non-invasive blood vessels, and BALF was performed with 2.0 $\mathrm{ml}$ pre-cooled normal saline (0.9\%). The lavage was repeated three times, and it was considered successful if the recovery rate was above $80 \%$. Finally, the total protein content in the BALF was measured using Bicinchoninic acid (BCA) Protein Quantitation Kit (Solarbio, China) according to the manufacturer's protocol.

\section{Lung wet/dry ratio (W/D)}

Rats were sacrificed when the experimental period was complete. The left lung was isolated and weighed immediately (wet weight) after the lung surface liquid was absorbed using an absorbent paper. To obtain the dry weight, lungs were placed in a $60^{\circ} \mathrm{C}$ oven for $72 \mathrm{~h}$ until a constant weight was achieved. The lung wet/dry weight ratio was then calculated.

\section{Hematoxylin-eosin (H\&E) staining}

The middle lobe of the left lung was fixed with $4 \%$ paraformaldehyde. After fixation and dehydration, the specimens were embedded in paraffin and cut into $5 \mathrm{~mm}$-thick sections. Next, sections were stained with hematoxylin and eosin in accordance with routine procedures of hematoxylin-eosin staining, followed by observation of the histopathological changes of lungs under a light microscope (Olympus, Japan). Two pathologists then observed and scored the extent of lung tissue damage using the double-blind method. The lung tissue was scored according to the following four items: congestion/edema in the alveolar cavity; lung tissue hemorrhage and thickening; inflammatory cell infiltration in the alveolar cavity and blood vessel cavity; and alveolar wall congestion, edema, thickening, and hyaline membrane form [18]. The scores of the four aspects were added up to form a visual field lung injury score: 0 points for no 
pathological changes, 1 point for mild pathological changes, 2 points for moderate pathological changes, 3 points for severe pathological changes, and 4 points for very severe pathological changes. Finally, the 200 alveoli of lung tissue samples were examined at $\times 400$ magnification to determine the lung injury score.

\section{Immunohistochemistry (IHC)}

Sections were placed in the $60^{\circ} \mathrm{C}$ ovens to bake for $60 \mathrm{~min}$. After dewaxing and rehydrating, antigen retrieval was performed with $10 \mathrm{mM}$ citrate buffer (ZSGB-BIO, China) for $5 \mathrm{~min}$. Next, sections were incubated with the rabbit anti-rat MPO (1:2000 dilution, Abcam, USA) primary antibody overnight at $4{ }^{\circ} \mathrm{C}$. On the next day, the sections were incubated with the biotinylated sheep anti-rabbit IgG secondary antibody at room temperature for $20 \mathrm{~min}$, followed by staining with DAB from the SP-POD kit (ZSGB-BIO, China). Finally, sections were sealed with neutral resin and analyzed under a light microscope (Olympus, Japan). MPO-positive nuclei were counted in three randomly selected areas per section from each individual rat, and the percentage of MPO-positive nuclei was calculated for each area.

\section{Enzyme-linked immunosorbent assay (ELISA)}

BALF, serum, and lung specimens were used to evaluate the immunomodulatory function of the sedative. The cervical venous blood was collected and centrifuged at $3500 \mathrm{rpm} / \mathrm{min}$ for $15 \mathrm{~min}$ at $4^{\circ} \mathrm{C}$. The supernatant was then collected and stored at $-80^{\circ} \mathrm{C}$ until use. Next, lung tissues were homogenized and the supernatant was collected for analysis by ELISA. The level of TNF- $a$ and IL- $1 b$ in different specimens was determined using the ELISA kit (Shanghai Enzyme-linked Biotechnology Co. Ltd., China) according to the manufacturer's instructions.

\section{Statistical analysis}

All statistical analyses were performed using SPSS statistical software 22.0 (SPSS Inc, Chicago, IL, USA). Measurement data were expressed as mean \pm standard deviation. Repeated measurement analysis of variance (ANOVA) was used to compare indexes at different time points in the same group, whereas one-way ANOVA was used to compare indexes at the same time points among different groups. $P<0.05$ was considered statistically significant.

\section{Results}

\section{Sevoflurane improved oxygenation function and stabilized circulation}

Table 1 summarizes the blood gas analysis (BGA) and hemodynamic variables at each stage. Results showed that there were no significant differences between the two groups with regard to $\mathrm{pH}(P>0.05)$. There were significant differences in $\mathrm{PaO}_{2}, \mathrm{PaCO}_{2}, \mathrm{PaO}_{2} / \mathrm{FiO}_{2}$, and MAP in the two groups at the $\mathrm{T} 1$ stage compared to the T0 stage $(P<0.05)$, suggesting successful establishment of the ARDS rat model. Similarly, there were also significant differences in $\mathrm{PaO}_{2}, \mathrm{PaCO}_{2}, \mathrm{PaO}_{2} / \mathrm{FiO}_{2}$, and MAP between the two groups in the T2 stage compared to the T1 stage $(P<0.05)$, indicating that VV ECMO was beneficial to 
ARDS in rats. At the T2 stage, the $\mathrm{PaO}_{2}$ and $\mathrm{PaO}_{2} / \mathrm{FiO}_{2}$ between the Sevo and Pro groups were statistically significant $(P<0.05)$, suggesting that sevoflurane could improve oxygenation.

Table 1

Important physiological parameters of rats at each stage

\begin{tabular}{|c|c|c|c|c|}
\hline Main parameter & Group & TO & $\mathrm{T} 1$ & $\mathrm{~T} 2$ \\
\hline \multirow[t]{2}{*}{$\mathrm{pH}$} & Sevo & $7.292 \pm 0.095$ & $7.276 \pm 0.049$ & $7.266 \pm 0.026$ \\
\hline & Pro & $7.317 \pm 0.100$ & $7.267 \pm 0.020$ & $7.251 \pm 0.034$ \\
\hline \multirow[t]{2}{*}{$\mathrm{PaO}_{2}(\mathrm{mmHg})$} & Sevo & $164.4 \pm 8.0$ & $99.8 \pm 8.3^{\ddagger}$ & $346.8 \pm 9.4^{* \dagger}$ \\
\hline & Pro & $165.2 \pm 8.7$ & $98.4 \pm 7.0^{\ddagger}$ & $312.6 \pm 10.4^{\dagger}$ \\
\hline \multirow[t]{2}{*}{$\mathrm{PaCO}_{2}(\mathrm{mmHg})$} & Sevo & $37.2 \pm 6.1$ & $49.2 \pm 3.8^{\ddagger}$ & $44.0 \pm 9.0^{\dagger}$ \\
\hline & Pro & $38.2 \pm 7.2$ & $48.8 \pm 3.8^{\ddagger}$ & $45.0 \pm 8.7^{\dagger}$ \\
\hline \multirow[t]{2}{*}{$\mathrm{PaO}_{2} / \mathrm{FiO}_{2}$} & Sevo & $427.0 \pm 10.4$ & $262.6 \pm 8.8^{\ddagger}$ & $383.6 \pm 7.9 * \dagger$ \\
\hline & Pro & $428.4 \pm 10.5$ & $263.6 \pm 5.3^{\ddagger}$ & $353.4 \pm 8.7^{\dagger}$ \\
\hline \multirow[t]{2}{*}{ MAP (mmHg) } & Sevo & $111.2 \pm 8.4$ & $58.4 \pm 3.9^{\ddagger}$ & $90.0 \pm 7.5^{\dagger}$ \\
\hline & Pro & $113.0 \pm 8.0$ & $60.0 \pm 3.2^{\ddagger}$ & $81.8 \pm 6.3^{\dagger}$ \\
\hline \multicolumn{5}{|c|}{$* p<0.05$ vs Pro; ${ }^{\dagger} p<0.05$ vs T $1 ;{ }^{\ddagger} p<0.05$ vs T0 } \\
\hline \multicolumn{5}{|c|}{$\begin{array}{l}\text { Abbreviations: Sevo: Sevoflurane group; Pro: propofol group; T0: Baseline; } \mathrm{T} 1 \text { : The time to ARDS; T2: } \\
\text { Weaning from } \mathrm{ECMO} \text { for } 1 \mathrm{~h} ; \mathrm{PaO}_{2} \text { : arterial partial pressure of oxygen; } \mathrm{PaCO}_{2} \text { : arterial partial pressure } \\
\text { of carbon dioxide; } \mathrm{PaO}_{2} / \mathrm{FiO}_{2} \text { : oxygenation index; MAP: mean arterial pressure. }\end{array}$} \\
\hline
\end{tabular}

\section{Sevoflurane alleviated lung injury during VV ECMO-assisted ARDS in rats}

$\mathrm{HE}$ staining revealed the pathological changes of lung tissues in the two groups. The pathologic changes in the Pro group (Fig. 2a) were evident, and lung tissues showed mild interstitial hyperplasia, moderate vascular congestion, and moderate inflammatory cell infiltration. Results showed that mild pulmonary interstitial hyperplasia and vasocongestion were scattered in the Sevo group (Fig. 2b), and inflammatory cell infiltration was significantly less than in the Pro group. Fig. 2c shows the lung injury scores. Moreover, the W/D ratio of the Pro group was significantly increased compared to the Sevo group $(P<0.05$; Fig. 3a). With regard to the BALF protein content (Fig. 3b), it was found that the Sevo group could significantly reduce pulmonary capillary alveolar leakage compared to the Pro group $(P<0.001)$.

Sevoflurane inhibited the release of inflammatory mediators and decreased neutrophil infiltration in lung tissue during WV ECMO-assisted ARDS in rats 
Myeloperoxidase (MPO) staining in lung tissues of the Sevo group and Pro group are shown in Fig. 4. The positive expression of MPO was significantly lower in the Sevo group compared to the Pro group $(P<0.05$; Fig. 4c). Fig. 5 shows the BALF, lung, and serum levels of TNF- $\alpha$ and IL-1 $\beta$ in rats. The levels of TNF- $\alpha$ and $\mathrm{IL}-1 \beta$ in the Pro group were higher than in the Sevo group $(P<0.05)$, indicating that sevoflurane could relieve inflammatory responses in ARDS rats.

\section{Discussion}

This study found that sevoflurane was significantly better than propofol in improving oxygenation function and reducing inflammation during VV ECMO. Evidence suggests that ARDS is one of the most common complex acute diseases in the clinic, with a fatality rate of about 30\% [19]. At present, VV ECMO is a clinically accepted technique for temporarily supporting ARDS patients who do not respond to conventional treatment to achieve a "lung rest strategy" $[20,21]$. The inflammatory process in ARDS is caused by the release of inflammatory mediators by pulmonary endothelial cells, macrophages, and neutrophils [22]. During this process, the microvascular barrier of alveolar tissue is destroyed, and inflammatory cells from the blood vessels accumulate in the lung tissue [23]. Notably, the hyaline membrane is formed when inflammatory cells accumulate, thereby making it difficult to transfer oxygen from alveolar tissue to blood vessels [24].

In recent years, several studies have confirmed that sedative drugs can reduce lung inflammation during the sedation of ARDS patients $[25,26]$. Sevoflurane is a commonly used inhalation anesthetic whose antiinflammatory properties have been demonstrated in LPS-induced lung injury, whereas propofol is a commonly used intravenous anesthetic in clinical practice [27]. Studies have revealed that the protective effect of propofol on lung tissue is associated with oxidative stress and inflammatory response. Specifically, propofol can scavenge hydroxyl free radicals and $\mathrm{H}_{2} \mathrm{O}_{2}$, induce $\mathrm{HO}-1$ expression, and inhibit the expression of IL-6, TNF-a, and other inflammatory factors, thereby playing a protective role on organs [28-30]. This study found that different sedatives produce different anti-inflammatory effects during VV ECMO-assisted ARDS, with sevoflurane being significantly superior to propofol.

Furthermore, neutrophils and macrophages were involved in the inflammatory response of ARDS, Cytokines such as IL-1 $\beta$ and TNF- $\alpha$ released by macrophages act locally through stimulating activation of chemotaxis and neutrophils [31]. It should be noted that neutrophils play an essential role in developing ARDS, and MPO is a marker protein of neutrophils [32]. Herein, results showed that sevoflurane significantly reduced the infiltration of pulmonary neutrophils during VV-ECMO-assisted ARDS. Previous experimental studies have shown that reducing inflammation of the lungs could improve pulmonary oxygenation function and pulmonary edema [27]. Our results showed that rats in the sevoflurane treatment group had less lung tissue pathological damage and lower W/D ratio compared to rats in the propofol group, suggesting that sevoflurane was superior to propofol in improving the oxygenation function and hemodynamic stability. It is worth mentioning that the protein concentration in BALF is an essential indicator of the structural integrity of the alveolar wall [33]. This study found that sevoflurane significantly reduced the protein concentration in BALF compared to propofol. 
However, this study had the following limitations: (1) the duration of the experiment was $3 \mathrm{~h}$ which only allowed observation of the early auxiliary effect on VV ECMO. Therefore, further studies should be conducted to explore the long-term effect. (2) We only used blood pressure, heart rate, and other simple indicators for anesthesia depth monitoring. (3) Although it was found that sevoflurane could improve oxygenation function and reduce inflammation, we did not explore the specific mechanism of action. Therefore, our next study will elucidate the underlying mechanism of action.

\section{Conclusions}

This study shows that sevoflurane sedation had a superior effect to propofol sedation in reducing lung injury, and improving lung inflammation and oxygenation in VV ECMO-assisted ARDS in rats, which laid a foundation for the subsequent molecular mechanism study.

\section{Abbreviations}

VV ECMO: Venovenous extracorporeal membrane oxygenation; OA: oleic acid; ARDS: Acute respiratory distress syndrome; BALF: bronchial alveolar lavage fluid; BCA: Bicinchoninic acid assay; Sevo: Sevoflurane; Pro: Propofol; MPO: Myeloperoxidase; MAP: mean arterial pressure; IHC: immunohistochemistry; ELISA: Enzyme-linked immunosorbent assay; IL-1ß: Interleukin 1 beta; TNFa:tumor necrosis factor Alpha; SD: Sprague-Dawley; PEEP: positive end-expiratory pressure; BGA: arterial blood gas analysis; W/D: wet/dry ratio; H\&E: Hematoxylin-eosin; ANOVA: Analysis of variance

\section{Declarations}

\section{Acknowledgements}

The authors acknowledge the Lanzhou University Second Hospital Laboratory Animal Center for providing the housing and breeding locations. All schematic diagrams were created with biorender.com.

\section{Authors' contributions}

RZZ designed and carried out all experiments, and wrote the manuscript. JH was involved in data analysis and preparation of figures. JBY, XDC, KRZ, SLW, and JL reviewed the manuscript. YNL and BRG supervised all work presented in this manuscript. All authors reviewed this manuscript. All data were generated in-house, and no paper mill was used. All authors agree to be accountable for all aspects of work, thereby ensuring integrity and accuracy.

\section{Funding}

This work was supported by the Cuiying Scientific and Technological Innovation Program of Lanzhou University Second Hospital [CY2019-QN01]; the Natural Science Foundation of Gansu Province, China 
[20JR10RA760, 20JR10RA745, 20JR10RA733]; and the Lanzhou University Second Hospital Talent introduction program in the hospital [YJRCKYQDJ-2021-02].

\section{Availability of data and materials}

The datasets used and/or analyzed during the current study are available from the corresponding author upon reasonable request.

\section{Ethics approval and consent to participate}

The study was performed in accordance with the animal care guidelines of the National Institutes of Health and Animal Research: Reporting of In Vivo Experiments (ARRIVE) guidelines. In addition, the study was approved by the institutional animal experimental ethics committee of Lanzhou University Second Hospital (Lanzhou, Gansu, China; approval number D2021-020)

\section{Consent for publication}

Not applicable.

\section{Competing interests}

The authors declare no competing interests.

\section{References}

1. Seethala RR, Hou PC, Aisiku IP, et al. Early risk factors and the role of fluid administration in developing acute respiratory distress syndrome in septic patients. Ann Intensive Care. 2017;7(1):11.

2. Ni L, Chen Q, Zhu K, et al. The influence of extracorporeal membrane oxygenation therapy on intestinal mucosal barrier in a porcine model for post-traumatic acute respiratory distress syndrome. J Cardiothorac Surg. 2015;10:20.

3. Sylvestre A, Adda M, Maltese F, et al. Long-term neurocognitive outcome is not worsened by of the use of venovenous ECMO in severe ARDS patients. Ann Intensive Care. 2019;9(1):82.

4. Langer $\mathrm{T}$, Santini A, Bottino N, et al. "Awake" extracorporeal membrane oxygenation (ECMO): pathophysiology, technical considerations, and clinical pioneering. Crit Care. 2016;20(1):150.

5. Xie Y, Cao L, Qian Y, et al. Effect of Deep Sedation on Mechanical Power in Moderate to Severe Acute Respiratory Distress Syndrome: A Prospective Self-Control Study. Biomed Res Int. 2020;2020:2729354.

6. Fuller BM, Roberts BW, Mohr NM, et al. A study protocol for a multicentre, prospective, before-andafter trial evaluating the feasibility of implementing targeted SEDation after initiation of mechanical ventilation in the emergency department (The ED-SED Pilot Trial). BMJ Open. 2020;10(12):e041987.

7. Ren $X, A i Y$, Zhang $L$, et al. Sedation and analgesia requirements during venovenous extracorporeal membrane oxygenation in acute respiratory distress syndrome patients. Perfusion. 
2021:2676591211052160.

8. Reddy HG, Maynes EJ, Saxena A, et al. Utilization of extracorporeal life support for diffuse alveolar damage and diffuse alveolar hemorrhage: A systematic review. Artif Organs. 2021;45(6):559-68.

9. Lin TY, Fang YF, Huang SH, et al. Capnography monitoring the hypoventilation during the induction of bronchoscopic sedation: A randomized controlled trial. Sci Rep. 2017;7(1):8685.

10. Walsh TS, Kydonaki K, Antonelli J, et al. Rationale, design and methodology of a trial evaluating three strategies designed to improve sedation quality in intensive care units (DESIST study). BMJ Open. 2016;6(3):e010148.

11. Montes RG, Bohn RA. Deep sedation with inhaled sevoflurane for pediatric outpatient gastrointestinal endoscopy. J Pediatr Gastroenterol Nutr. 2000;31(1):41-46.

12. Yang Z, Cheng F, Yan G, et al. Propofol protects against endotoxin-induced myocardial injury by inhibiting NF-kB-mediated inflammation. Exp Ther Med. 2018;15(2):2032-36.

13. Jia L, Hao H, Wang $\mathrm{C}$, et al. Etomidate attenuates hyperoxia-induced acute lung injury in mice by modulating the Nrf2/HO-1 signaling pathway. Exp Ther Med. 2021;22(1):785.

14. Jin Y, Zhao X, Li H, et al. Effects of sevoflurane and propofol on the inflammatory response and pulmonary function of perioperative patients with one-lung ventilation. Exp Ther Med. 2013;6(3):781-85.

15. Kwak SH, Choi Jl, Park JT. Effects of propofol on endotoxin-induced acute lung injury in rabbit. J Korean Med Sci. 2004;19(1):55-61.

16. Ferrando C, Aguilar G, Piqueras L, et al. Sevoflurane, but not propofol, reduces the lung inflammatory response and improves oxygenation in an acute respiratory distress syndrome model: a randomised laboratory study. Eur J Anaesthesiol. 2013;30(8):455-63.

17. Li Y, Huang J, Zhang R, et al. Establishment of a venovenous extracorporeal membrane oxygenation in a rat model of acute respiratory distress syndrome. Perfusion. 2021:2676591211031468.

18. Yan Z, Luo H, Xie B, et al. Targeting adaptor protein SLP76 of RAGE as a therapeutic approach for lethal sepsis. Nat Commun. 2021;12(1):308.

19. Ding $\mathrm{D}$, Xu S, Zhang H, et al. 3-Methyladenine and dexmedetomidine reverse lipopolysaccharideinduced acute lung injury through the inhibition of inflammation and autophagy. Exp Ther Med. 2018;15(4):3516-22.

20. Erdem Ö, Kuiper JW, van Rosmalen J, et al. The Sublingual Microcirculation Throughout Neonatal and Pediatric Extracorporeal Membrane Oxygenation Treatment: Is It Altered by Systemic Extracorporeal Support? Front Pediatr. 2019;7:272.

21. Nakatsutsumi K, Sekiya K, Urushibata N, et al. A successful case of extracorporeal membrane oxygenation treatment for intractable pneumothorax in a patient with COVID-19. Acute Med Surg. 2020;7(1):e612.

22. Chen QH, Wu F, Liu L, et al. Mesenchymal stem cells regulate the Th17/Treg cell balance partly through hepatocyte growth factor in vitro. Stem Cell Res Ther. 2020;11(1):91. 
23. Negrin LL, Halat G, Kettner S, et al. Club cell protein 16 and cytokeratin fragment 21-1 as early predictors of pulmonary complications in polytraumatized patients with severe chest trauma. PLoS One. 2017;12(4):e0175303.

24. Lee T, Packiriswamy N, Lee E, et al. Role of G protein-coupled receptor kinase-6 in Escherichia coli lung infection model in mice. Physiol Genomics. 2017;49(11):682-89.

25. Nieuwenhuijs-Moeke GJ, Jainandunsing JS, Struys M. Sevoflurane, a sigh of relief in COVID-19? Br J Anaesth. 2020;125(2):118-21.

26. Hu AM, Zhong XX, Li Z, et al. Comparative Effectiveness of Midazolam, Propofol, and Dexmedetomidine in Patients With or at Risk for Acute Respiratory Distress Syndrome: A Propensity Score-Matched Cohort Study. Front Pharmacol. 2021;12:614465.

27. Kellner P, Müller M, Piegeler T, et al. Sevoflurane Abolishes Oxygenation Impairment in a Long-Term Rat Model of Acute Lung Injury. Anesth Analg. 2017;124(1):194-203.

28. Gülçin I, Alici HA, Cesur M. Determination of in vitro antioxidant and radical scavenging activities of propofol. Chem Pharm Bull (Tokyo). 2005;53(3):281-85.

29. Kobayashi K, Yoshino F, Takahashi SS, et al. Direct assessments of the antioxidant effects of propofol medium chain triglyceride/long chain triglyceride on the brain of stroke-prone spontaneously hypertensive rats using electron spin resonance spectroscopy. Anesthesiology. 2008;109(3):426-35.

30. Xu JJ, Wang YL. Propofol attenuation of hydrogen peroxide-mediated oxidative stress and apoptosis in cultured cardiomyocytes involves haeme oxygenase-1. Eur J Anaesthesiol. 2008;25(5):395-402.

31. Ware LB, Matthay MA. The acute respiratory distress syndrome. N Engl J Med. 2000;342(18):133449.

32. Zhang ZT, Zhang DY, Xie K, et al. Luteolin activates Tregs to promote IL-10 expression and alleviating caspase-11-dependent pyroptosis in sepsis-induced lung injury. Int Immunopharmacol. 2021;99:107914.

33. Chung IS, Kim JA, Kim JA, et al. Reactive oxygen species by isoflurane mediates inhibition of nuclear factor KB activation in lipopolysaccharide-induced acute inflammation of the lung. Anesth Analg. 2013;116(2):327-35.

\section{Figures}




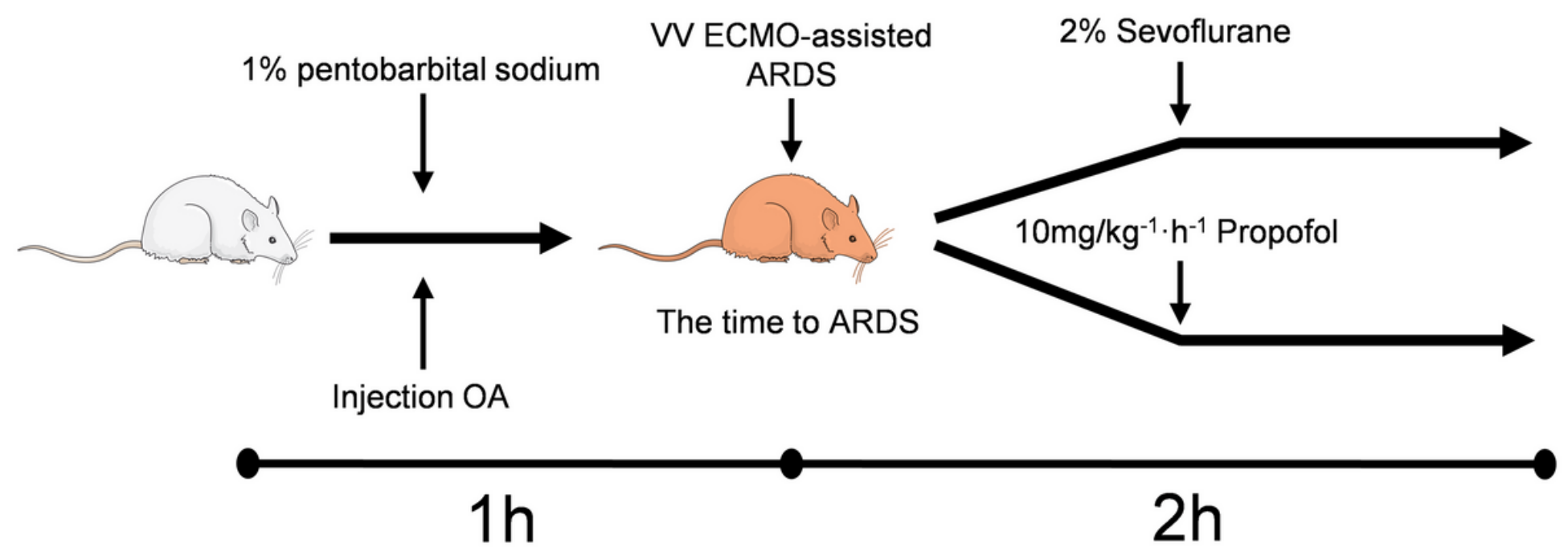

Figure 1

Flow diagram showing the experimental protocol. The experiment lasted for $3 \mathrm{~h}$, including $1 \mathrm{~h}$ of $\mathrm{OA}$ induced ARDS and $2 \mathrm{~h}$ of VV ECMO assisted ARDS. Created with BioRender (https://biorender.com/).

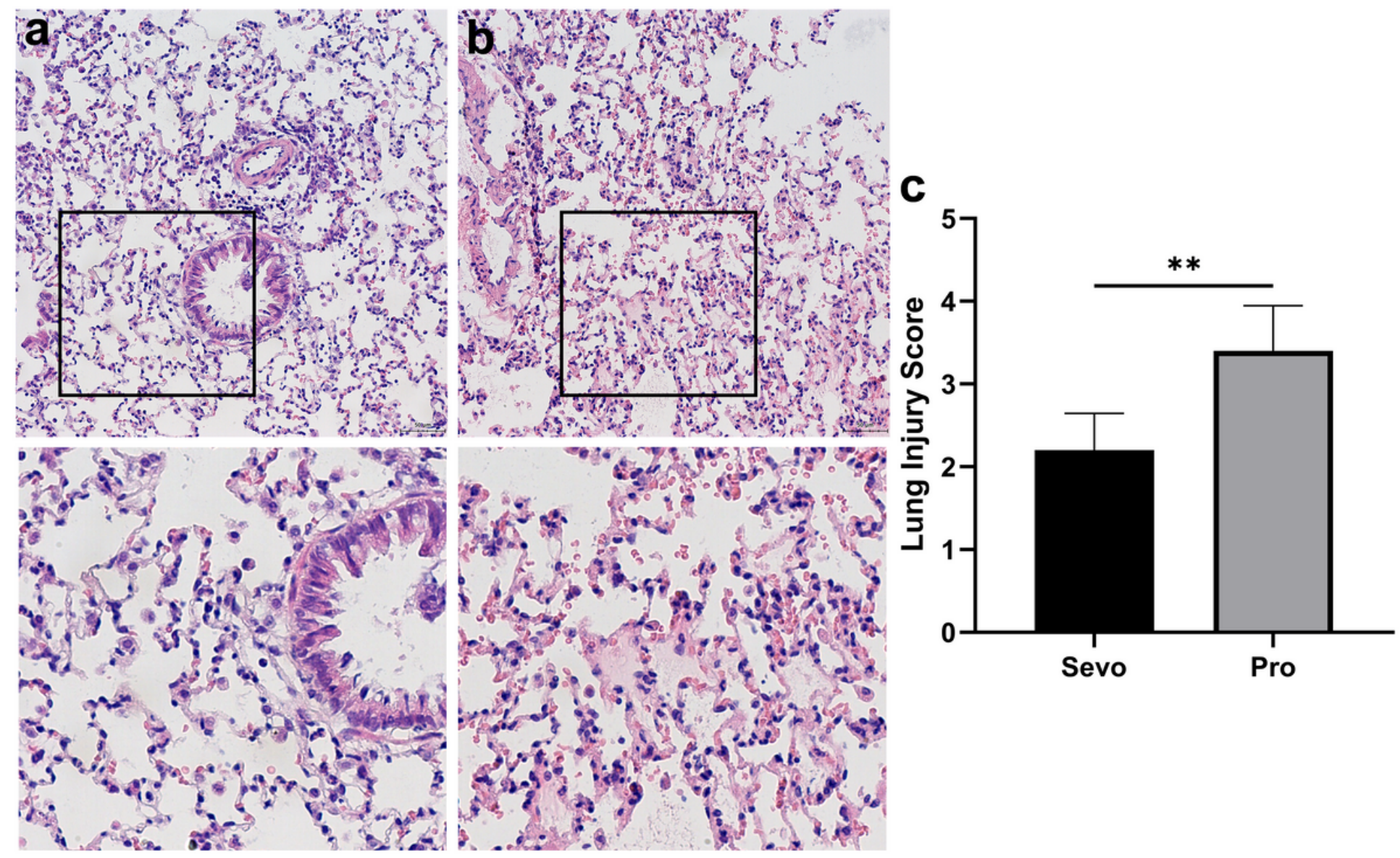

Figure 2

Pathological evaluation of lung tissues. Representative images of lung sections stained with

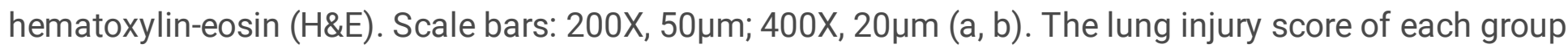


was graded from 0 (no injury) to 4 (maximum injury) (c). ${ }^{*} \mathrm{P}<0.01$, compared to Sevo group, $\mathrm{n}=12$ in each group.
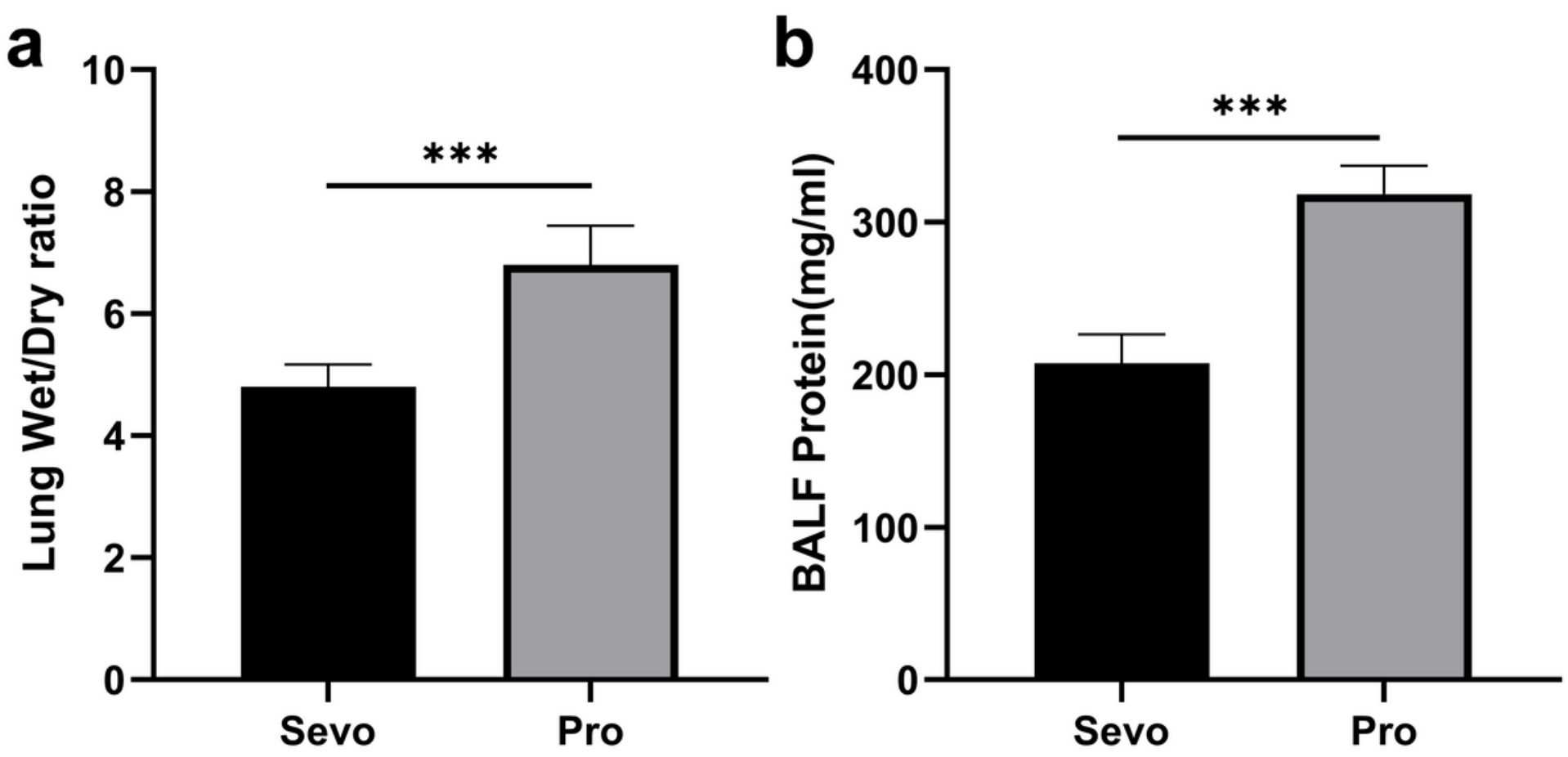

Figure 3

The water content in lung tissue was determined using the wet/dry ratio (a). The alveolar capillary barrier was determined by measuring the total protein content in BALF (b). ${ }^{\star \star *} \mathrm{P}<0.001$, compared to Sevo group, $n=12$ in each group. 


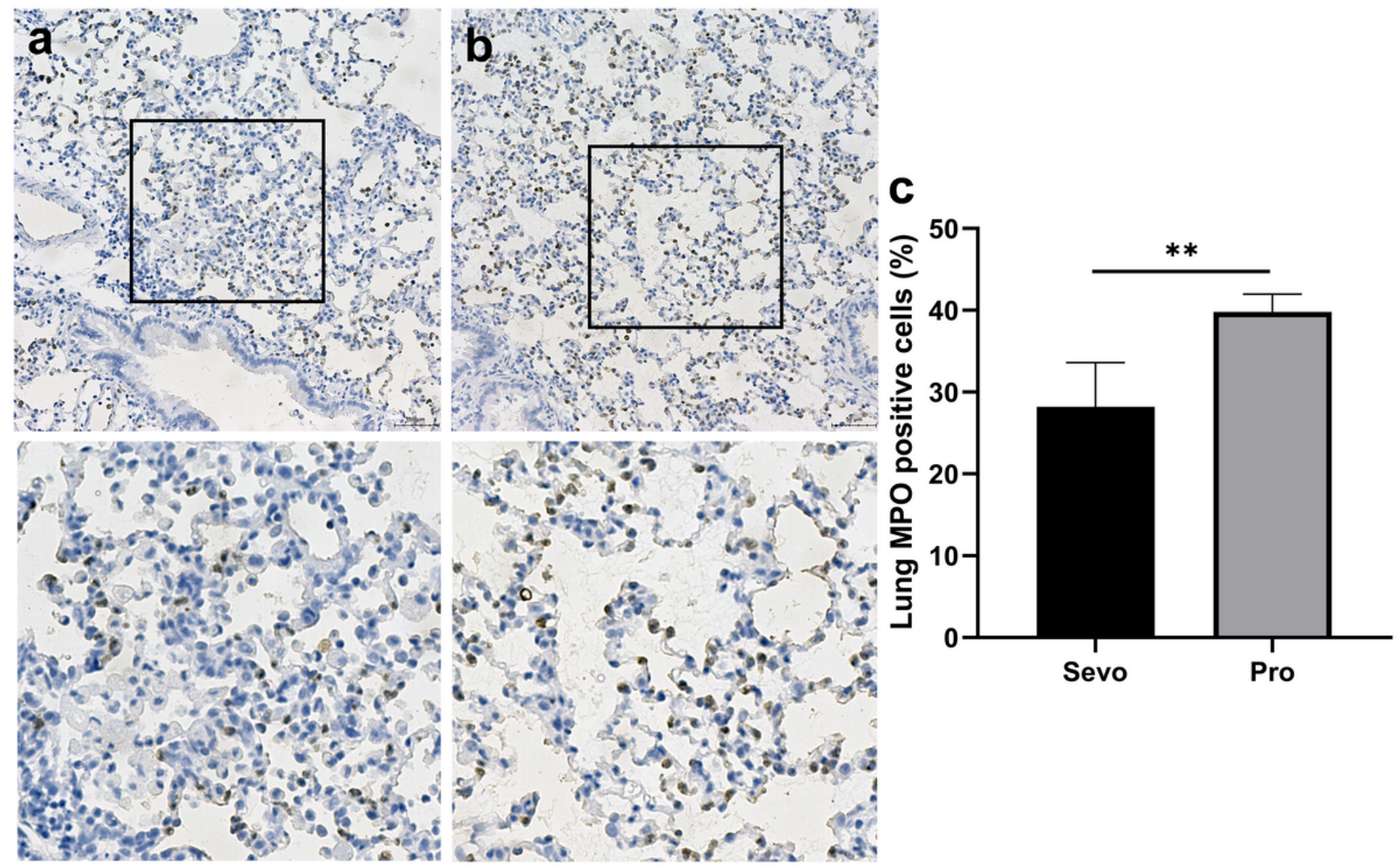

Figure 4

MPO staining was used to detect neutrophil infiltration in lung tissue. IHC staining was used to detect

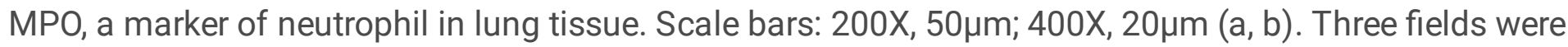
randomly selected to quantitatively analyze the activity of MPO (c). ${ }^{\star *} \mathrm{P}<0.01$, compared to Sevo group, $\mathrm{n}=12$ in each group. 

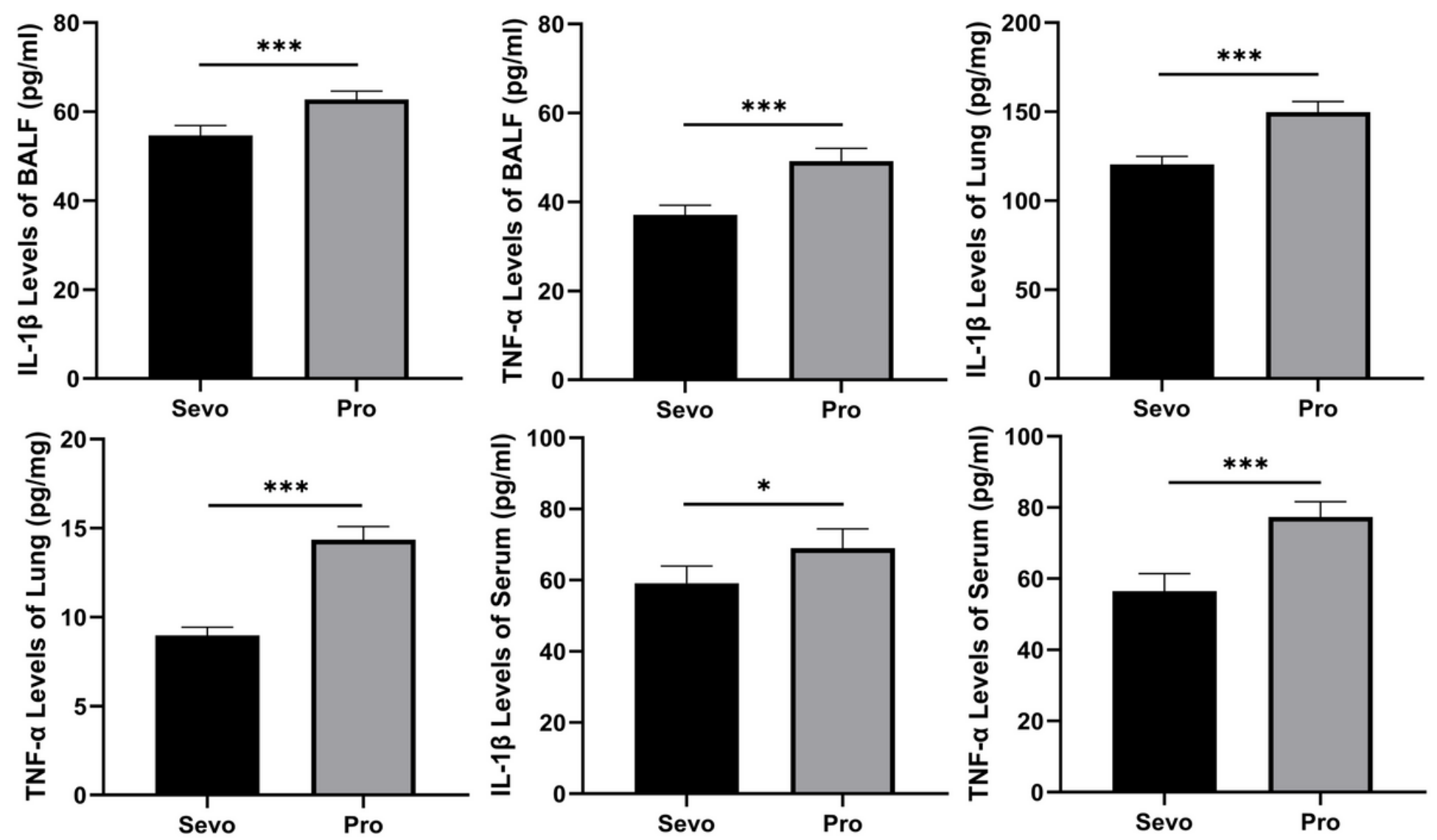

Figure 5

BALF, lung, and serum levels of TNF- $\alpha$ and IL- $1 \beta$ in each group of rats. The levels of IL-1 $\beta$ and TNF- $\alpha$ were decreased in BALF, lung, and serum of Sevo group rats compared to the Pro group rats. ${ }^{*} P<0.05$, $\star \star \star P<0.001$, compared with Sevo group, $n=12$ in each group. 